\title{
ABSOLUTE CONTINUITY OF MAPPINGS WITH FINITE GEOMETRIC DISTORTION
}

\author{
Ville Tengvall \\ University of Jyväskylä, Department of Mathematics and Statistics \\ P. O. Box 35 (MaD), FI-40014 University of Jyväskylä, Finland; ville.tengvall@jyu.fi
}

\begin{abstract}
Suppose that $\Omega \subset \mathbf{R}^{n}$ is a domain with $n \geq 2$. We show that a continuous, sense-preserving, open and discrete mapping of finite geometric outer distortion with $\mathcal{K}_{O}(\cdot, f) \in$ $L_{\text {loc }}^{1 /(n-1)}(\Omega)$ is absolutely continuous on almost every line parallel to the coordinate axes. Moreover, if $U \subset \Omega$ is an open set with $N(f, U)<\infty$, then $f$ satisfies the distortion inequality
\end{abstract}

$$
|D f(x)|^{n} \leq C|J(x, f)| \mathcal{K}_{O}(x, f)
$$

for almost every $x \in U$, where the constant $C>0$ depends only on $n$ and on the maximum multiplicity $N(f, U)$.

\section{Introduction}

Suppose that $\Omega \subset \mathbf{R}^{n}, n \geq 2$, is a domain. We recall that if $\omega: \Omega \rightarrow[0, \infty]$ is a measurable function, then the $\omega$-weighted (conformal) modulus of a family $\Gamma$ of paths in $\Omega$ is defined as

$$
\mathrm{M}_{\omega}(\Gamma)=\inf _{\rho \in \operatorname{Adm} \Gamma} \int_{\Omega} \rho^{n}(x) \omega(x) \mathrm{d} x .
$$

Above, a Borel function $\rho: \mathbf{R}^{n} \rightarrow[0, \infty]$ is admissible for $\Gamma$, abbr. $\rho \in \operatorname{Adm} \Gamma$, if for the line integral of $\rho$ along every path $\gamma \in \Gamma$ we have

$$
\int_{\gamma} \rho(x)|\mathrm{d} x| \geq 1
$$

Furthermore, when $\omega \equiv 1$, we write $\mathrm{M}(\Gamma)$ instead of $\mathrm{M}_{1}(\Gamma)$.

We call a mapping $f: \Omega \rightarrow \mathbf{R}^{n}$

(i) open if it takes open sets in $\Omega$ to open sets in $\mathbf{R}^{n}$,

(ii) discrete if the set $f^{-1}(y)$ of preimages is a discrete set for every poin $y \in \mathbf{R}^{n}$, and

(iii) if $f$ is continuous, we say that it is sense-preserving if for the local degree of a mapping $f$ we have $\operatorname{deg}(y, f, D)>0$ for every subdomain $D \subset \subset \Omega$ and for all points $y \in f(D) \backslash f(\partial D)$. For more details we refer the reader to [18, I.4].

Mapping $f: \Omega \rightarrow \Omega^{\prime}$ between two domains $\Omega, \Omega^{\prime} \subset \mathbf{R}^{n}, n \geq 2$, is called quasiconformal if

$(\mathrm{QC}-1) f$ is a sense-preserving homeomorphism, and

doi:10.5186/aasfm.2015.4018

2010 Mathematics Subject Classification: Primary 30C62, 30C65.

Key words: Mappings of finite distortion, moduli inequalities, $Q$-mappings.

The author was supported by the Academy of Finland, and by the Vilho, Yrjö and Kalle Väisälä foundation. Part of this research was done while the author was visiting at the University of Michigan. He would like to thank the department for its hospitality. 
(QC-2) there exists constants $\mathcal{K}_{O}(f) \in[1, \infty)$ and $\mathcal{K}_{I}(f) \in[1, \infty)$ such that the two-sided moduli inequality

$$
\mathrm{M}_{1 / \mathcal{K}_{O}(f)}(\Gamma) \leq \mathrm{M}(f(\Gamma)) \leq \mathrm{M}_{\mathcal{K}_{I}(f)}(\Gamma)
$$

holds for all path families $\Gamma$ in $\Omega$.

Similarly, we call a mapping $f: \Omega \rightarrow \mathbf{R}^{n}$ quasiregular if

(QR-1) $f$ is a continuous, sense-preserving, discrete and open mapping, and

(QR-2) there exists constants $\mathcal{K}_{O}(f) \in[1, \infty)$ and $\mathcal{K}_{I}(f) \in[1, \infty)$ such that the two-sided moduli inequality

$$
N(f, A)^{-1} \mathrm{M}_{1 / \mathcal{K}_{O}(f)}(\Gamma) \leq \mathrm{M}(f(\Gamma)) \leq \mathrm{M}_{\mathcal{K}_{I}(f)}(\Gamma)
$$

holds for all path families $\Gamma$ in $A$ and for every Borel set $A \subset \Omega$ with $N(f, A):=\sup _{y \in \mathbf{R}^{n}}$ card $f^{-1}(y) \cap A<\infty$.

These two definitions are called geometric definitions of quasiconformal and quasiregular mappings. Even though quasiconformal mappings are not always assumed to be sense-preserving in the geometric definition, it is convenient to require this, if we want to consider quasiregular mappings as generalized quasiconformal mappings. For more details about quasiconformal and quasiregular mappings we refer to $[17,18,23]$.

In order to study a larger class of mappings we may relax the topological and geometrical assumptions in the definitions of quasiconformal and quasiregular mappings. This leads us to define mappings with finite geometric distortion.

Definition 1.1. Let $f: \Omega \rightarrow \mathbf{R}^{n}$ be a continuous mapping.

(i) Mapping $f$ is called a mapping of finite geometric outer distortion if there exists a measurable function $\mathcal{K}_{O}(\cdot, f): \Omega \rightarrow[1, \infty]$ taking finite values almost everywhere and satisfying

$$
\mathrm{M}_{1 / \mathcal{K}_{O}(\cdot, f)}(\Gamma) \leq C_{O}(A) \mathrm{M}(f(\Gamma))
$$

for every Borel set $A \subset \Omega$ for which $N(f, A)<\infty$, for every family of paths $\Gamma$ in $A$, and for some positive and finite constant $C_{O}(A)$ depending only on $n$ and $N(f, A)$. A function $\mathcal{K}_{O}(\cdot, f)$ which satisfies (3) is called outer geometric distortion function of $f$.

(ii) Mapping $f$ is called a mapping of finite geometric inner distortion if there exists a measurable function $\mathcal{K}_{I}(\cdot, f): \Omega \rightarrow[1, \infty]$ taking finite values almost everywhere and satisfying

$$
\mathrm{M}(f(\Gamma)) \leq C_{I}(A) \mathrm{M}_{\mathcal{K}_{I}(\cdot, f)}(\Gamma)
$$

for every Borel set $A \subset \Omega$ for which $N(f, A)<\infty$, for every family of paths $\Gamma$ in $A$, and for some positive and finite constant $C_{I}(A)$ depending only on $n$ and $N(f, A)$. A function $\mathcal{K}_{I}(\cdot, f)$ which satisfies (4) is called inner geometric distortion function of $f$.

The motivation for our study of mappings of finite geometric distortion comes from the study of mappings of finite distortion. We say that a mapping $f: \Omega \rightarrow \mathbf{R}^{n}$ is a mapping of finite (analytic) distortion if

(FD-1) $f \in W_{\text {loc }}^{1,1}\left(\Omega, \mathbf{R}^{n}\right)$,

(FD-2) $J(\cdot, f)$ is locally integrable, and 
(FD-3) there is a measurable function $K_{O}(\cdot, f): \Omega \rightarrow[1, \infty]$, finite almost everywhere, such that $f$ satisfies the classical distortion inequality

$$
|D f(x)|^{n} \leq K_{O}(x, f) J(x, f)
$$

for almost every $x \in \Omega$.

Above, $|D f(x)|$ is the operator norm of the differential matrix $D f(x)$ and $J(x, f)$ $=\operatorname{det} D f(x)$ is the pointwise Jacobian determinant of $f$.

From the analytical point of view mappings of finite distortion can be considered as generalizations of quasiconformal and quasiregular mappings. Moreover, by requiring some integrability for the distortion function $K_{O}(\cdot, f)$ we may conclude that in several occasions these mappings still satisfy many of the good properties of quasiconformal and quasiregular mappings, see $[4,6]$. Our interest is to see how closely mappings of finite (analytic) distortion and mappings of finite geometric distortion are related.

Sense-preserving mappings of finite geometric inner distortion with $C_{I} \equiv 1$ are usually called $Q$-mappings and they have been studied by several authors. To mention some of the basic properties of these mappings we remark that Salimov and Sevost'yanov [21, 22], see also [20], have shown that every continuous, open and discrete $Q$-mapping $f$ with $\mathcal{K}_{I}(\cdot, f) \in L_{\text {loc }}^{1}(\Omega)$

(i) is differentiable almost everywhere,

(ii) belongs to the Sobolev space $W_{\text {loc }}^{1,1}\left(\Omega, \mathbf{R}^{n}\right)$, and

(iii) satisfy the distortion inequality

$$
|D f(x)|^{n} \leq C(n) \mathcal{K}_{I}^{n-1}(x, f)|J(x, f)|
$$

for almost every $x \in \Omega$, where the constant $C(n)>0$ depends only on the dimension.

In addition, by applying [18, Lemma I.4.11] we see that these mappings are actually mappings of finite distortion. Similar kind of properties can be easily obtained also for sense-preserving mappings of finite geometric inner distortion with an arbitrary constant $C_{I}>0$. For more details about $Q$-mappings we refer to the works by Martio, Ryazanov, Srebro and Yakubov [13]-[17], and their references.

The less studied class out of these two geometric mapping classes is mappings with finite geometric outer distortion. It is important to note that lower moduli estimates similar to (3) have found signifigant applications to the theory of the Beltrami equations $[8,11]$ and the mappings of Orlicz-Sobolev classes $[9,10]$.

To give some examples of these mappings, we see that every quasiconformal and quasiregular mapping is a mapping of finite geometric outer distortion. Also every continuous, open and discrete mapping $f \in W_{\text {loc }}^{1, n}\left(\Omega, \mathbf{R}^{n}\right)$ of finite distortion is a mapping of finite geometric outer distortion. One can see this by using Väisälä's differentiability result [24], see also [17, Lemma 2.6], and then use same kind of argumentation as in [18, Theorem II.2.4].

In this paper we focus on studying under which conditions mappings of geometric outer distortion are mappings of finite distortion. The main result of this paper is the following theorem.

Theorem 1.2. Suppose that $\Omega \subset \mathbf{R}^{n}$ is a domain with $n \geq 2$. Let $f: \Omega \rightarrow \mathbf{R}^{n}$ be a continuous, sense-preserving, discrete and open mapping with finite geometric 
outer distortion $\mathcal{K}_{O}(\cdot, f) \in L_{\text {loc }}^{1 /(n-1)}(\Omega)$. Then $f \in W_{\text {loc }}^{1,1}\left(\Omega, \mathbf{R}^{n}\right)$. Moreover, if $U \subset \Omega$ is an open set with $N(f, U)<\infty$, then $f$ satisfies the distortion inequality

$$
|D f(x)|^{n} \leq C|J(x, f)| \mathcal{K}_{O}(x, f)
$$

for almost every $x \in U$, where $C:=C_{O}(U) N(f, U)$.

Some of the arguments in the proof of Theorem 1.2 go back to the papers by Koskela and Rogovin [7], and Martio, Rickman and Väisälä [12]. Similar kind of techiques are also used in [1].

One should notice that under the assumptions of Theorem 1.2 we do not know if $f$ is differentiable almost everywhere, which causes some difficulties. For instance, the non-negativity of the Jacobian is not known in all cases. However, if $n \in\{2,3\}$ then it follows from [5, Theorem 1.1] that every sense-preserving homeomorphism $f \in W_{\text {loc }}^{1,1}\left(\Omega, \mathbf{R}^{n}\right)$ has non-negative Jacobian at almost every point. On the other hand, it follows from [4, Theorem A. 35] that under the assumptions of Theorem 1.2 we always have $J(\cdot, f) \in L_{\text {loc }}^{1}(\Omega)$. These two facts gives us the following corollary of Theorem 1.2.

Corollary 1.3. Suppose that $\Omega, \Omega^{\prime} \subset \mathbf{R}^{n}$ are domains with, and $n \in\{2,3\}$. Let $f: \Omega \rightarrow \Omega^{\prime}$ be a sense-preserving homomorphism of finite geomeric outer distortion with $\mathcal{K}_{O}(\cdot, f) \in L_{\text {loc }}^{1 /(n-1)}(\Omega)$. Then $f$ is a mapping of finite distortion.

\section{Absolute continuity on lines}

In this section we will show that if $f: \Omega \rightarrow \mathbf{R}^{n}$ is a mapping of finite geometric distortion with $\mathcal{K}_{O}(\cdot, f) \in L_{\text {loc }}^{1 /(n-1)}(\Omega)$, then it is absolutely continuous on almost every line segment parallel to the coordinate axes in $\Omega$, abbr. $f \in \operatorname{ACL}\left(\Omega, \mathbf{R}^{n}\right)$. Especially, we will show that $f \in W_{\text {loc }}^{1,1}\left(\Omega, \mathbf{R}^{n}\right)$. However, before going to proofs of these results, let us recall the definition and some basic properties of quasiadditive set functions.

Suppose that $U \subset \mathbf{R}^{n}$ is an open set, and denote by $\operatorname{Bor}(U)$ the set of all Borel subsets of $U$. A mapping $\psi: \operatorname{Bor}(U) \rightarrow[0, \infty]$ is called m-quasiadditive set function, $m \geq 1$, if the following conditions are satisfied for all Borel sets in $U$ :

(i) $A \subset B$ implies $\psi(A) \leq \psi(B)$.

(ii) $\psi(A)<\infty$ if $A$ is a compact set.

(iii) If $A_{1}, \ldots, A_{k}$ are disjoint and if $A_{i} \subset A$, then

$$
\sum_{i=1}^{k} \psi\left(A_{k}\right) \leq m \psi(A)
$$

We define upper and lower derivatives of a $m$-quasiadditive set function $\psi$ at a point $x \in U$ as

$$
\bar{\psi}^{\prime}(x)=\limsup _{r \rightarrow 0} \frac{\psi\left(B^{n}(x, r)\right)}{\mathrm{m}_{n}\left(B^{n}(x, r)\right)} \quad \text { and } \quad \underline{\psi}^{\prime}(x)=\liminf _{r \rightarrow 0} \frac{\psi\left(B^{n}(x, r)\right)}{\mathrm{m}_{n}\left(B^{n}(x, r)\right)},
$$

where $\mathrm{m}_{k}(A)$ denotes the $k$-dimensional Lebesgue measure of a set $A$, and $B^{k}(x, r)$ is the open ball $\left\{y \in \mathbf{R}^{k}:|x-y|<r\right\}$.

It is well-known that $m$-quasiadditive set function $\psi: \operatorname{Bor}(U) \rightarrow[0, \infty]$ satisfies the following properties:

(qa1) $\bar{\psi}^{\prime}$ and $\underline{\psi}^{\prime}$ are Borel functions, 
(qa2) $\bar{\psi}^{\prime}(x) \leq m \psi^{\prime}(x)<\infty$ for almost every $x \in U$, and

(qa3) for each open set $V \subset U$ we have

$$
\int_{V} \underline{\psi}^{\prime}(x) \mathrm{d} x \leq m \psi(V) \text {. }
$$

One can proof the conditions (qa1)-(qa2) similarly as the corresponding results are proved for Borel measures in [2]. Henceforth we will refer to these properties by (qa1), (qa2) and (qa3). For more details we refer the reader to [12].

In the proof of following proposition we will use the idea of Koskela and Rogovin [7, Theorem 1.1].

Proposition 2.1. Let $\Omega \subset \mathbf{R}^{n}$ be a domain with $n \geq 2$. Let $f: \Omega \rightarrow \mathbf{R}^{n}$ be a continuous, sense-preserving, discrete and open mapping with finite geometric distortion $\mathcal{K}_{O}(\cdot, f) \in L_{\text {loc }}^{1 /(n-1)}(\Omega)$. Then $f \in \operatorname{ACL}\left(\Omega, \mathbf{R}^{n}\right)$.

Proof. Pick a closed cube $Q \subset \subset \Omega$ the sides of which are parallel to the coordinate axes. Then by [18, Proposition 4.10] we have $m:=N(f, Q)<\infty$.

Assume that $Q=Q_{0} \times J_{0}$, where $Q_{0}$ is $(n-1)$-interval in $\mathbf{R}^{n-1}$, and $J_{0}=[a, b] \subset$ R. By symmetry it suffices to show that $f$ is absolutely continuous on almost every line segment in $Q$ parallel to the $x_{n}$-axis.

For $A \in \operatorname{Bor}\left(Q_{0}\right)$ write

$$
\Phi(A):=\mathrm{m}_{n}(f(A \times[a-d, b+d])) \leq \mathrm{m}_{n}\left(f\left(Q_{d}\right)\right)<\infty,
$$

where $d=\frac{1}{10} \operatorname{dist}(Q, \partial \Omega)$ and $Q_{d}:=\{x \in \Omega: \operatorname{dist}(x, Q) \leq d\}$. Then $\Phi$ is a $m$ quasiadditive set funtion on $Q_{0}$ and thus by (qa2) we have

$$
\bar{\Phi}^{\prime}(x):=\limsup _{r \rightarrow 0} \frac{\Phi\left(B^{n-1}(x, r)\right)}{\mathrm{m}_{n-1}\left(B^{n-1}(x, r)\right)}<\infty
$$

for almost every $x \in Q_{0}$. Denote $E_{0}:=\left\{x \in Q_{0}: \bar{\Phi}^{\prime}(x)\right.$ is not finite $\}$.

Next, consider the set

$$
\begin{aligned}
\mathcal{A}:=\{ & I \subset J_{0}: I \text { is a finite union of closed intervals, whose interiors } \\
& \text { are mutually disjoint and whose end points are rational }\} .
\end{aligned}
$$

Observe that this set is countable.

Now, for almost every $y \in Q_{0}$ we know by Fubini's theorem that

$$
\int_{\{y\} \times[a-d, b+d]} \mathcal{K}_{O}^{\frac{1}{n-1}}(y, z ; f) \mathrm{d} z<\infty .
$$

Denote by $E_{1}$ the set where (8) fails. Let us define for every $I \in \mathcal{A}$ a function $g_{I}: Q_{0} \rightarrow \overline{\mathbf{R}}$ by

$$
g_{I}(y):=\int_{\{y\} \times I} \mathcal{K}_{O}^{\frac{1}{n-1}}(y, z ; f) \mathrm{d} z
$$

By Fubini's theorem $g_{I} \in L^{1}\left(Q_{0}\right)$ and thus for almost every $y \in Q_{0}$

$$
\lim _{r \rightarrow 0} f_{B^{n-1}(y, r)} g_{I}(x) \mathrm{d} x=g_{I}(y) .
$$


Denote by $E_{I}$ the set where this is not true. Now the set

$$
E=E_{0} \cup E_{1} \cup\left(\bigcup_{I \in \mathcal{A}} E_{I}\right)
$$

has measure zero, because it is a countable union of sets of measure zero.

Fix $y \in Q_{0} \backslash E$. It suffices to show that $f$ is absolutely continuous on the segment $\{y\} \times J_{0}$. Let $\left\{I_{i}\right\}_{i=1}^{l}, I_{i}=\left[a_{i}, b_{i}\right]$, be a union of closed intervals on $J_{0}$ whose interiors are mutually disjoint, and whose endpoints are rational numbers. Because our goal is to estimate the sum $\sum_{i=1}^{l}\left|f\left(y, a_{i}\right)-f\left(y, b_{i}\right)\right|$ from above, we may assume that

$$
\left|f\left(y, a_{i}\right)-f\left(y, b_{i}\right)\right| \neq 0 \quad \text { for every } i=1, \ldots, l .
$$

Since $f$ is continuous, for every $i=1, \ldots, l$ there is $\delta_{i}>0$ such that

$$
\left|f\left(y, a_{i}\right)-f(x)\right|<\frac{\left|f\left(y, a_{i}\right)-f\left(y, b_{i}\right)\right|}{4}, \quad \text { when }\left|\left(y, a_{i}\right)-x\right|<\delta_{i}
$$

and

$$
\left|f\left(y, b_{i}\right)-f(x)\right|<\frac{\left|f\left(y, a_{i}\right)-f\left(y, b_{i}\right)\right|}{4}, \quad \text { when }\left|\left(y, b_{i}\right)-x\right|<\delta_{i} .
$$

Denote $\delta=\min _{i} \delta_{i}$. Let $0<r<\frac{1}{10} \min \left\{\delta,\left|a_{i}-b_{i}\right|, d\right\}$. We cover the set $\{y\} \times$ $\left(\bigcup_{i=1}^{l} I_{i}\right)$ by cylinders $C_{i}=B(y, r) \times\left(a_{i}-\epsilon, b_{i}+\epsilon\right)$. We may choose $\epsilon>0$ to be so small that

$$
B^{n-1}(y, r / 2) \times\left(\bigcup_{i} I_{i}\right) \subset \bigcup_{i} C_{i} \subset B^{n-1}(y, 2 r) \times[a-d, b+d] .
$$

Define

$$
\tilde{\rho}_{i}(y)=2\left|f\left(y, a_{i}\right)-f\left(y, b_{i}\right)\right|^{-1} \chi_{f\left(C_{i}\right)}(y), \quad i=1, \ldots, l,
$$

and denote $G_{i}:=\left|f\left(y, a_{i}\right)-f\left(y, b_{i}\right)\right|$. We define the path family $\Gamma_{i}$ by

$$
\Gamma_{i}=\left\{\gamma_{x}^{i}: x \in B^{n-1}(y, r / 2)\right\}
$$

where $\gamma_{x}^{i}:\left[a_{i}, b_{i}\right] \rightarrow \mathbf{R}^{n}$ is defined as $\gamma_{x}^{i}(t)=(x, t)$.

Suppose that $\gamma \in \Gamma_{i}$, for some $i=1, \ldots, l$, and $f \circ \gamma$ is rectifiable. Then for the line integral over path $f \circ \gamma$ we have

$$
\int_{f \circ \gamma} \tilde{\rho}_{i} \mathrm{~d} s=2 G_{i}^{-1} \int_{f \circ \gamma} \chi_{f\left(C_{i}\right)} \mathrm{d} s \geq G_{i}^{-1}\left|f\left(y, a_{i}\right)-f\left(y, b_{i}\right)\right|=1 .
$$

This implies that $\tilde{\rho}_{i}$ is an admissible test function for the modulus $\mathrm{M}\left(f\left(\Gamma_{i}\right)\right)$. By calculating, we have

$$
\int_{\mathbf{R}^{n}} \tilde{\rho}_{i}^{n}(y) \mathrm{d} y=2^{n} G_{i}^{-n} \int_{\mathbf{R}^{n}} \chi_{f\left(C_{i}\right)}(y)^{n} \mathrm{~d} y \leq 2^{n} G_{i}^{-n} \mathrm{~m}_{n}\left(f\left(C_{i}\right)\right) .
$$

This implies

$$
\mathrm{M}_{1 / \mathcal{K}_{O}}\left(\Gamma_{i}\right) \leq C_{O} \mathrm{M}\left(f\left(\Gamma_{i}\right)\right) \leq 2^{n} C_{O} G_{i}^{-n} \mathrm{~m}_{n}\left(f\left(C_{i}\right)\right),
$$

where the finite constants $C_{O}>0$ depends only on $n$ and $m$. 
On the other hand, if $\gamma_{x}^{i} \in \Gamma_{i}, x \in B^{n-1}(y, r)$, and $\rho_{i}$ is an arbitrary test function for the modulus $\mathrm{M}_{1 / \mathcal{K}_{O}}\left(\Gamma_{i}\right)$, we have

$$
\begin{aligned}
& \alpha_{n-1} r^{n-1} \leq \int_{B^{n-1}(y, r)} \int_{\gamma_{x}^{i}} \rho_{i}(x, z)|\mathrm{d} z| \mathrm{dm}_{n-1}(x) \\
& \leq \int_{B^{n-1}(y, r)}\left(\int_{\gamma_{x}^{i}} \rho_{i}^{n}(x, z) \frac{1}{\mathcal{K}_{O}(x, z ; f)}|\mathrm{d} z|\right)^{\frac{1}{n}}\left(\int_{\gamma_{x}^{i}} \mathcal{K}_{O}^{\frac{1}{n-1}}(x, z ; f)|\mathrm{d} z|\right)^{\frac{n-1}{n}} \mathrm{dm}_{n-1}(x) \\
& \leq\left(\int_{B^{n-1}(y, r)} \int_{\gamma_{x}^{i}} \rho_{i}^{n}(x, z) \frac{1}{\mathcal{K}_{O}(x, z ; f)}|\mathrm{d} z| \mathrm{dm}_{n-1}(x)\right)^{\frac{1}{n}} \\
& \cdot\left(\int_{B^{n-1}(y, r)} \int_{\gamma_{x}^{i}} \mathcal{K}_{O}^{\frac{1}{n-1}}(x, z ; f)|\mathrm{d} z| \mathrm{dm}_{n-1}(x)\right)^{\frac{n-1}{n}},
\end{aligned}
$$

where $\alpha_{n-1}$ is the volume of an $(n-1)$-dimensional unit ball. Combining this with (9), we see that

$$
\begin{aligned}
& \left|f\left(y, a_{i}\right)-f\left(y, b_{i}\right)\right| \\
& \leq C\left(\frac{\mathrm{m}_{n}\left(f\left(C_{i}\right)\right)}{r^{n-1} \alpha_{n-1}}\right)^{\frac{1}{n}}\left(f_{B^{n-1}(y, r)} \int_{\gamma_{x}^{i}} \mathcal{K}_{O}^{\frac{1}{n-1}}(x, z ; f)|\mathrm{d} z| \mathrm{dm}_{n-1}(x)\right)^{\frac{n-1}{n}} \\
& =C\left(\frac{\mathrm{m}_{n}\left(f\left(C_{i}\right)\right)}{r^{n-1} \alpha_{n-1}}\right)^{\frac{1}{n}}\left(f_{B^{n-1}(y, r)} \int_{I_{i}} \mathcal{K}_{O}^{\frac{1}{n-1}}(x, t ; f) \mathrm{d} t \mathrm{dm}_{n-1}(x)\right)^{\frac{n-1}{n}}
\end{aligned}
$$

where $C:=2 C_{O}^{1 / n}$. Here on the third line we have used [23, Theorem 4.1] and the fact that $\left|\left(\gamma_{x}^{i}\right)^{\prime}(t)\right|=1$.

By summing over $i=1, \ldots, l$ and using Hölder's inequality, we get

$$
\begin{aligned}
& \sum_{i=1}^{l}\left|f\left(y, a_{i}\right)-f\left(y, b_{i}\right)\right| \\
& \leq C \sum_{i=1}^{l}\left[\left(\frac{\mathrm{m}_{n}\left(f\left(C_{i}\right)\right)}{r^{n-1} \alpha_{n-1}}\right)^{\frac{1}{n}}\left(f_{B^{n-1}(y, r)} \int_{I_{i}} \mathcal{K}_{O}^{\frac{1}{n-1}}(x, t ; f) \mathrm{d} t \mathrm{dm}_{n-1}(x)\right)^{\frac{n-1}{n}}\right] \\
& =C\left(\sum_{i=1}^{l} \frac{\mathrm{m}_{n}\left(f\left(C_{i}\right)\right)}{r^{n-1} \alpha_{n-1}}\right)^{\frac{1}{n}}\left(\sum_{i=1}^{l} f_{B^{n-1}(y, r)} \int_{I_{i}} \mathcal{K}_{O}^{\frac{1}{n-1}}(x, t ; f) \mathrm{d} t \mathrm{dm}_{n-1}(x)\right)^{\frac{n-1}{n}} \\
& \leq C\left(\frac{\Phi\left(B^{n-1}(y, 2 r)\right)}{r^{n-1} \alpha_{n-1}}\right)^{\frac{1}{n}}\left(f_{B^{n-1}(y, r)} \int_{\cup_{i=1}^{l} I_{i}} \mathcal{K}_{O}^{\frac{1}{n-1}}(x, t ; f) \mathrm{d} t \mathrm{dm}_{n-1}(x)\right)^{\frac{n-1}{n}}
\end{aligned}
$$

with $C=2 C_{O}^{1 / n}$. Taking upper limit with respect to $r$ on both sides, we arrive at

$$
\sum_{i=1}^{l}\left|f\left(y, a_{i}\right)-f\left(y, b_{i}\right)\right| \leq C \bar{\Phi}^{\prime}(y)^{\frac{1}{n}}\left(\int_{\cup_{i=1}^{l} I_{i}} \mathcal{K}_{O}^{\frac{1}{n-1}}(y, t ; f) \mathrm{d} t\right)^{\frac{n-1}{n}}
$$

with $C=2 C_{O}^{1 / n}$. This holds for rational $a_{i}, b_{i}$. By continuity, it then holds for all $a_{i}, b_{i} \in \mathbf{R}$. Thus $f$ is absolutely continuous on $\{y\} \times J_{0}$.

The proof of the following lemma is originally from [12, Lemma 2.17] with only small technical changes. However, to convince a reader we will go trough the proof. 
Lemma 2.2. Suppose that $\Omega \subset \mathbf{R}^{n}$ is a domain with $n \geq 2$. Let $f: \Omega \rightarrow \mathbf{R}^{n}$ be a continuous, sense-preserving, discrete and open mapping of finite geometric distortion with $\mathcal{K}_{O}(\cdot, f) \in L_{\text {loc }}^{1 /(n-1)}(\Omega)$. Then $f \in W_{\text {loc }}^{1,1}\left(\Omega, \mathbf{R}^{n}\right)$.

Proof. To prove that $f \in W_{\text {loc }}^{1,1}\left(\Omega, \mathbf{R}^{n}\right)$ it is enough to show the local integrability of the partial derivatives, whose existence is guaranteed by the ACL-property given by Proposition 2.1 .

Let $Q \subset \subset \Omega$ be an open $n$-interval. Then by [18, Proposition 4.10] we have $m:=N(f, Q)<\infty$. Choose an integer $k_{0}$ such that $0<1 / k_{0}<\operatorname{dist}(Q, \partial \Omega)$. We define

$$
h(x)=\left|\partial_{n} f(x)\right| \quad \text { and } \quad h_{k}(x)=\frac{k}{2} \int_{-\frac{1}{k}}^{\frac{1}{k}} h\left(x+t e_{n}\right) \mathrm{d} t,
$$

where $e_{n}$ stands for the $n$th standard basis vector of $\mathbf{R}^{n}$. Then $h_{k}(x)$ is defined for almost every $x \in Q$ and for all $k \geq k_{0}$.

First we show that $h_{k} \rightarrow h$ almost everywhere in $Q$ as $k \rightarrow \infty$. It is well known that $h$ is measurable. In fact, $h$ is a Borel function, see [19, p. 170]. Hence the function $(x, t) \mapsto h\left(x+t e_{n}\right)$ is measurable in $Q \times(-1 / k, 1 / k)$. By Fubini's theorem, this implies that $h_{k}$ is measurable.

Write $Q=Q_{0} \times J_{0}$, where $Q_{0}$ is an open $(n-1)$-interval and $J_{0}=(a, b)$. Then for almost every $z \in Q_{0}$ the mapping $t \mapsto f(z, t)$ is absolutely continuous with respect to $t \in\left(a-1 / k_{0}, b+1 / k_{0}\right)$. For such $z$ Lebesgue's differentiation theorem implies that $h_{k}(z, t) \rightarrow h(z, t)$ for almost every $t \in J_{0}$ as $k \rightarrow \infty$. Thus by Fubini's theorem it follows that $\liminf _{k \rightarrow \infty} h_{k}(x)=h(x)=\limsup _{k \rightarrow \infty} h_{k}(x)$ a.e. in $Q$. Therefore $h_{k} \rightarrow h$ almost everywhere in $Q$ as $k \rightarrow \infty$.

Next, by Fubini's theorem almost every $\alpha \in J_{0}$ has the property that $h_{k}(z, \alpha) \rightarrow$ $h(z, \alpha)$ for almost every $z \in Q_{0}$. Consider $\alpha$ with this property, and set

$$
F_{k}(A):=\mathrm{m}_{n}(f(A \times(\alpha-1 / k, \alpha+1 / k)))
$$

for all Borel sets $A \subset Q_{0}$ and each $k \geq k_{0}$. Then the set functions $F_{k}$ are $m$ quasiadditive set functions on $Q_{0}$. By similar argument than in the Proposition 2.1 we see that for almost every $z \in Q_{0}$ the function $t \mapsto f(z, t)$ is absolutely continuous on $[\alpha-1 / k, \alpha+1 / k]$ and that its total variation is not greater than

$$
C\left(\bar{F}_{k}^{\prime}(z)\right)^{\frac{1}{n}}\left(\int_{\alpha-1 / k}^{\alpha+1 / k} \mathcal{K}_{O}^{\frac{1}{n-1}}(z, t) \mathrm{d} t\right)^{\frac{n-1}{n}}
$$

where $C>0$ is some finite constant depending only on $n$ and $m$. Consequently

$$
h_{k}(z, \alpha)=\frac{k}{2} \int_{\alpha-1 / k}^{\alpha+1 / k}\left|\partial_{n} f(z, t)\right| \mathrm{d} t \leq C \frac{k}{2}\left(\bar{F}_{k}^{\prime}(z)\right)^{\frac{1}{n}}\left(\int_{\alpha-1 / k}^{\alpha+1 / k} \mathcal{K}_{O}^{\frac{1}{n-1}}(z, t) \mathrm{d} t\right)^{\frac{n-1}{n}}
$$

with a finite constant $C>0$ depending only on $n$ and $m$. By integrating over $z \in Q_{0}$, using Hölder's inequality, and applying (qa2) and (qa3), we get

$$
\int_{Q_{0}} h_{k}(z, \alpha) \operatorname{dm}_{n-1} \leq C m^{\frac{2}{n}} \frac{k}{2} F_{k}\left(Q_{0}\right)^{\frac{1}{n}}\left(\int_{Q_{0}} \int_{\alpha-1 / k}^{\alpha+1 / k} \mathcal{K}_{O}^{\frac{1}{n-1}}(z, t) \mathrm{d} t \mathrm{dm}_{n-1}(z)\right)^{\frac{n-1}{n}}
$$




$$
\begin{gathered}
=C m^{\frac{2}{n}} \frac{k}{2} \mathrm{~m}_{n}\left(f\left(Q_{0} \times(\alpha-1 / k, \alpha+1 / k)\right)\right)^{\frac{1}{n}} \\
\cdot\left(\int_{Q_{0}} \int_{\alpha-1 / k}^{\alpha+1 / k} \mathcal{K}_{O}^{\frac{1}{n-1}}(z, t) \mathrm{d} t \mathrm{dm}_{n-1}(z)\right)^{\frac{n-1}{n}},
\end{gathered}
$$

where the finite constant $C>0$ depends only on $n$ and $m$. For each Borel set $A \subset J_{0}$ define

$$
\psi(A)=\mathrm{m}_{n}\left(f\left(Q_{0} \times A\right)\right)
$$

Then $\psi$ is a $m$-quasiadditive set function on $J_{0}$. As $k \rightarrow \infty$, (12) together with Fatou's lemma implies that

$$
\int_{Q_{0}} h(z, \alpha) \operatorname{dm}_{n-1}(z) \leq C m^{\frac{2}{n}}\left(\bar{\psi}^{\prime}(\alpha)\right)^{\frac{1}{n}}\left(\int_{Q_{0}} \mathcal{K}_{O}^{\frac{1}{n-1}}(z, \alpha) \operatorname{dm}_{n-1}(z)\right)^{\frac{n-1}{n}}
$$

for almost every $\alpha$. By integrating over $\alpha \in J_{0}$, using Hölder's inequality, and applying (qa2) and (qa3), it follows from (13) that

$$
\int_{Q} h \mathrm{dm}_{n} \leq C m^{\frac{4}{n}} \mathrm{~m}_{n}(f(Q))^{\frac{1}{n}}\left(\int_{Q} \mathcal{K}_{O}^{\frac{1}{n-1}}(z, \alpha) \mathrm{dm}_{n-1}(z)\right)^{\frac{n-1}{n}},
$$

where the constant $C>0$ is finite and depends only on $n$ and $m$. Thus $h$ is integrable over $Q$.

\section{Proof of the distortion inequality}

Our main result, Theorem 1.2, will follow directly from Lemma 2.2, Theorem 3.1 and Lemma 3.2. Therefore we give proofs only for Theorem 3.1 and Lemma 3.2, and leave rest of the proof for a reader.

Suppose that $f: \Omega \rightarrow \mathbf{R}^{n}$ is a sense-preserving, continuous, discrete and open mapping, and consider an open subset $U \subset \Omega$ such that $N(f, U)<\infty$. Then we define a $m$-quasiadditive set function $\mu(\cdot, f): \operatorname{Bor}(U) \rightarrow[0, \infty]$ by setting

$$
\mu(A, f)=\mathrm{m}_{n}(f(A))
$$

for each $A \in \operatorname{Bor}(U)$. Unlike in section 2, we will define upper and lower limits for this specific quasiadditive set functions as

$$
\bar{\mu}^{\prime}(x, f)=\limsup _{r \rightarrow 0} \frac{\mathrm{m}_{n}(f(Q(x, r)))}{\mathrm{m}_{n}(Q(x, r))} \quad \text { and } \quad \underline{\mu}^{\prime}(x, f)=\liminf _{r \rightarrow 0} \frac{\mathrm{m}_{n}(f(Q(x, r)))}{\mathrm{m}_{n}(Q(x, r))},
$$

where $Q(x, r):=\left\{y \in \mathbf{R}^{n}:\left|x_{i}-y_{i}\right|<r\right.$ for all $\left.i=1, \ldots, n\right\}$. Here $z_{i}$ stands for the $i$ th coordinate of a point $z \in \mathbf{R}^{n}$.

One can check that conditions (qa1)-(qa3) remains the same for the derivatives $\bar{\mu}^{\prime}(x, f)$ and $\underline{\mu}^{\prime}(x, f)$. This is because Vitali covering theorem holds also for the family of all closed cubes.

Theorem 3.1. Let $\Omega \subset \mathbf{R}^{n}$ be a domain with $n \geq 2$. Suppose that $f: \Omega \rightarrow \mathbf{R}^{n}$ is a continuous, sense-preserving, open and discrete mapping, and that there exists a function $\mathcal{K}_{O}(\cdot, f) \in L_{\text {loc }}^{1 /(n-1)}(\Omega)$ such that

$$
\mathrm{M}_{1 / \mathcal{K}_{O}(\cdot, f)}(\Gamma) \leq C_{O}(A) \mathrm{M}(f(\Gamma))
$$


for every Borel set $A \subset \Omega$ for which $N(f, A)<\infty$, for every family of paths $\Gamma$ in $A$, and for some positive and finite constant $C_{O}(A)$ depending only on $n$ and $N(f, A)$. Then $f \in W_{\text {loc }}^{1,1}\left(\Omega, \mathbf{R}^{n}\right)$. Moreover, if $U \subset \Omega$ is an open set with $N(f, U)<\infty$, then

$$
|D f(x)|^{n} \leq C_{O}(U) \mathcal{K}_{O}(x, f) \underline{\mu}^{\prime}(x, f)
$$

for almost every $x \in U$.

Proof. We have already shown that $f \in W_{\text {loc }}^{1,1}\left(\Omega, \mathbf{R}^{n}\right)$. Thus, by $[2$, Theorem 1 , p. 228] $f$ is $L^{1}$-differentiable, i.e.

$$
\lim _{r \rightarrow 0} \frac{1}{r} f_{B(x, r)}|f(y)-f(x)-D f(x)(y-x)| \mathrm{d} y=0 \quad \text { for a.e. } x \in \Omega .
$$

Given $\epsilon>0$, denote

$$
S_{r}^{\epsilon}(x)=\{y \in \Omega:|f(y)-f(x)-D f(x)(y-x)| \geq \epsilon r\} .
$$

Then $L^{1}$-differentiability gives that

$$
\lim _{r \rightarrow 0} \frac{\mathrm{m}_{n}\left(S_{r}^{\epsilon}(x)\right)}{\mathrm{m}_{n}(Q(x, r))}=0
$$

for almost every $x \in \Omega$.

Pick an open set $U \subset \Omega$ with $m:=N(f, U)<\infty$, and fix a point $x \in U$ such that

(i) (16) holds at $x$,

(ii) $x$ is a Lebesgue point of $\mathcal{K}_{O}^{\frac{1}{n-1}}(\cdot, f)$, and

(iii) $\bar{\mu}^{\prime}(x, f)$ is finite.

We observe that almost every point in $U$ satisfies the above criteria.

By applying translations we may assume that $x=0=f(x)$. Moreover, because every matrix $A$ can be written as $U D V^{T}$, where $U$ and $V$ are orthogonal and

$$
D=\left(\begin{array}{cccc}
\alpha_{1} & 0 & \cdots & 0 \\
0 & \alpha_{2} & \cdots & 0 \\
\vdots & \vdots & \ddots & \vdots \\
0 & 0 & \cdots & \alpha_{n}
\end{array}\right)
$$

where $\alpha_{1} \geq \alpha_{2} \geq \cdots \geq \alpha_{n} \geq 0$, we can assume that our $D f(0)$ is as above.

We have to show that

$$
\alpha_{1}^{n} \leq \mathcal{K}_{O}(0, f) \underline{\mu}^{\prime}(0, f) .
$$

We assume that $\alpha_{1}>0$ since otherwise the claim is trivial. Next, fix $0<\delta<\frac{1}{2}$ and $0<\epsilon \leq \min \left\{\alpha_{1} / 4, \delta / 2\right\}$. Choose $r_{\epsilon}>0$ such that $\mathrm{m}_{n}\left(S_{r}^{\epsilon}(x)\right) \leq \frac{\epsilon}{2} \mathrm{~m}_{n}(Q(x, r))$ whenever $r \leq r_{\epsilon}$. Fix $0<r \leq r_{\epsilon}$, and set

$$
E=[-r,-(1-\delta) r] \times[-r, r]^{n-1}, \quad F=[(1-\delta) r, r] \times[-r, r]^{n-1} \text { and } Q_{r}=[-r, r]^{n} \text {. }
$$

Denote $G_{E}=E \backslash S_{r}^{\epsilon}(0)$ and $G_{F}=F \backslash S_{r}^{\epsilon}(0)$. Let $\Gamma$ be the family of all curves that connects $G_{E}$ and $G_{F}$ in $Q_{r}$. Define $P: \mathbf{R}^{n} \rightarrow \mathbf{R}^{n-1}$ by setting

$$
P\left(y_{1}, \ldots, y_{n}\right)=\left(y_{2}, \ldots, y_{n}\right) \text {. }
$$


Notice that then $\mathrm{m}_{n-1}\left(P\left(G_{E}\right) \cap P\left(G_{F}\right)\right) \geq\left(1-\frac{\epsilon}{\delta}\right)(2 r)^{n-1}$. If $\rho$ is admissible for $\Gamma$, and if $J_{y}=[-r, r] \times\{y\}$, where $y \in P\left(G_{E}\right) \cap P\left(G_{F}\right)$, we have

$$
\int_{J_{y}} \rho(x)|\mathrm{d} x| \geq 1
$$

since a subcurve of $J_{y}$ belongs to $\Gamma$. Thus by Fubini and Hölder's inequality, we obtain

$$
\begin{aligned}
\left(1-\frac{\epsilon}{\delta}\right)(2 r)^{n-1} & \leq \int_{P\left(G_{E}\right) \cap P\left(G_{F}\right)} 1 \mathrm{dm}_{n-1} \leq \int_{Q_{r}} \rho(z) \mathrm{d} z \\
& \leq\left(\int_{Q_{r}} \frac{\rho(z)^{n}}{\mathcal{K}_{O}(z, f)} \mathrm{d} z\right)^{\frac{1}{n}}\left(\int_{Q_{r}} \mathcal{K}_{O}(z, f)^{\frac{1}{n-1}} \mathrm{~d} z\right)^{\frac{n-1}{n}}
\end{aligned}
$$

Since $\rho$ is an arbitrary test function, using our assumption (15) we have that

$$
\left(1-\frac{\epsilon}{\delta}\right)(2 r)^{n-1} \leq C_{O}(U)^{\frac{1}{n}}(\mathrm{M}(f(\Gamma)))^{\frac{1}{n}}\left(\int_{Q_{r}} \mathcal{K}_{O}(z, f)^{\frac{1}{n-1}} \mathrm{~d} z\right)^{\frac{n-1}{n}}
$$

Next, suppose that $\gamma \in \Gamma$. Then it follows from

$$
\begin{aligned}
& f\left(G_{E}\right) \subset\left[-\left(\alpha_{1}+\epsilon\right) r,-\left(\alpha_{1}(1-\delta)-\epsilon\right) r\right] \times \mathbf{R}^{n-1}, \text { and } \\
& f\left(G_{F}\right) \subset\left[\left(\alpha_{1}(1-\delta)-\epsilon\right) r,\left(\alpha_{1}+\epsilon\right) r\right] \times \mathbf{R}^{n-1}
\end{aligned}
$$

that length $(f(\gamma)) \geq 2\left(\alpha_{1}(1-\delta)-\epsilon\right) r$. Thus $\tilde{\rho}(x)=\left(2\left(\alpha_{1}(1-\delta)-\epsilon\right) r\right)^{-1} \chi_{f\left(Q_{r}\right)}(x)$ is admissible for $\mathrm{M}(f(\Gamma))$, and we get

$$
\mathrm{M}(f(\Gamma)) \leq \frac{\mathrm{m}_{n}\left(f\left(Q_{r}\right)\right)}{\left(2\left(\alpha_{1}(1-\delta)-\epsilon\right) r\right)^{n}} .
$$

Therefore, by combining (17) and (18) we have

$$
\left(1-\frac{\epsilon}{\delta}\right)\left(\alpha_{1}(1-\delta)-\epsilon\right) \leq C_{O}(U)^{\frac{1}{n}}\left(\frac{\mathrm{m}_{n}\left(f\left(Q_{r}\right)\right)}{\mathrm{m}_{n}\left(Q_{r}\right)}\right)^{\frac{1}{n}}\left(f_{Q_{r}} \mathcal{K}_{O}(z, f)^{\frac{1}{n-1}} \mathrm{~d} z\right)^{\frac{n-1}{n}}
$$

Taking lower limit $r \rightarrow 0$, then letting $\epsilon \rightarrow 0$, and finally $\delta \rightarrow 0$ we get

$$
\alpha_{1} \leq C_{O}(U)^{\frac{1}{n}} \mathcal{K}_{O}^{\frac{1}{n}}(0, f) \underline{\mu}^{\prime}(0, f)^{\frac{1}{n}}
$$

and the claim follows.

Lemma 3.2. Let $\Omega \subset \mathbf{R}^{n}$ be a domain with $n \geq 2$, and let $f \in W_{\mathrm{loc}}^{1,1}\left(\Omega, \mathbf{R}^{n}\right)$ be a continuous, discrete and open mapping. Let $U \subset \subset \Omega$ be an open set with $N(f, U)<\infty$. Then

$$
\underline{\mu}^{\prime}(x, f) \leq N(f, U)|J(x, f)|
$$

for almost every $x \in U$.

Proof. Let us denote $m:=N(f, U)<\infty$. By using (qa3) together with a standard approximation argument, we conclude that

$$
\int_{A} \underline{\mu^{\prime}}(x, f) \mathrm{d} x \leq m \mathrm{~m}_{n}(f(A))
$$

for each Borel set $A \subset U$. 
For simplicity let us suppose that $U=\Omega$. Because $f \in W_{\text {loc }}^{1,1}\left(\Omega, \mathbf{R}^{n}\right)$, we have by [4, Theorem A.31] that $f$ is approximatively differentiable almost everywhere, and by applying [4, Corollary A.27] we may decompose

$$
\Omega=N \cap \bigcup_{k=1}^{\infty} \Omega_{k}
$$

where $N$ is a Borel set with null measure, the sets $\Omega_{k}$ are measurable and pairwise disjoint, and the restriction map $\left.f\right|_{\Omega_{k}}$ to each set $\Omega_{k}$ is Lipschitz. Then by the Area formula for Lipschitz mappings we get

$$
\int_{\Omega_{k}}|J(x, f)| \mathrm{d} x=\int_{\mathbf{R}^{n}} N\left(y, f, \Omega_{k}\right) \mathrm{d} y
$$

on each set $\Omega_{k}$, where $N(y, f, A):=$ card $f^{-1}(y) \cap A$ is the multiplicity function of $f$ with respect to a set $A \subset \Omega$. Therefore

$$
\int_{\mathbf{R}^{n}} N(y, f, \Omega \backslash N) \mathrm{d} y=\sum_{k=1}^{\infty} \int_{\mathbf{R}^{n}} N\left(y, f, \Omega_{k}\right) \mathrm{d} y=\int_{\Omega \backslash N}|J(x, f)| \mathrm{d} x .
$$

Let us denote $\mathcal{N}:=\Omega \backslash N$. Then $\mathcal{N}$ is a Borel set of full measure in $\Omega$, and by using (qa3), (20) and (22) we get

$$
\begin{aligned}
& \int_{B_{r}} \underline{\mu^{\prime}}(z, f) \mathrm{d} z=\int_{B_{r} \cap \mathcal{N}} \underline{\mu^{\prime}}(z, f) \mathrm{d} z \leq m \mathrm{~m}_{n}\left(f\left(B_{r} \cap \mathcal{N}\right)\right)=m \int_{\mathbf{R}^{n}} \chi_{f\left(B_{r} \cap \mathcal{N}\right)}(y) \mathrm{d} y \\
& \leq m \int_{\mathbf{R}^{n}} N\left(y, f, B_{r} \cap \mathcal{N}\right) \mathrm{d} y=m \int_{B_{r} \cap \mathcal{N}}|J(z, f)| \mathrm{d} z=m \int_{B_{r}}|J(z, f)| \mathrm{d} z,
\end{aligned}
$$

where $B_{r}:=B(x, r)$. By using Lebesgue differentiation theorem we have

$$
\underline{\mu}^{\prime}(x, f) \leq m|J(x, f)|
$$

for almost every $x \in \Omega$.

Acknowledgement. The author would like to thank Jani Onninen and Kai Rajala for their many useful comments on the manuscript, and Aapo Kauranen and Ville Kirsilä for sharing their thoughts on this topic. The author would also like to thank the referees for their careful reading of the manuscript and for many useful suggestions which improved the presentation of the paper.

\section{References}

[1] Ainong, F.: The ACL property of homeomorphisms under weak conditions. - Acta Math. Sin. New Series 14:4, 1998, 473-480.

[2] Evans, L. C., and R. F. Gariepy: Measure theory and fine properties of functions. - CRC Press, Boca Raton, FL, 1992.

[3] Federer, H.: Geometric measure theory. - Grundlehren Math. Wiss. 153, Springer-Verlag New York Inc., New York, 1969.

[4] Hencl, S., and P. Koskela: Lectures on mappings of finite distortion. - Lecture Notes in Math. 2096, Springer, Cham, 2014.

[5] Hencl, S., and J. MALÝ: Jacobians of Sobolev homeomorphisms. - Calc. Var. Partial Differential Equations 38:1-2, 2010, 233-242.

[6] Iwaniec, T., and G. Martin: Geometric function theory and nonlinear analysis. - Clarendon Press, Oxford Univ. Press, 2001. 
[7] Koskela, P., and S. Rogovin: Linear dilatation and absolute continuity. - Ann. Acad. Sci. Fenn. Math. 30:2, 2005, 385-392.

[8] Kovtonyuk, D. A., I. V. Petkov, V. I. Ryazanov, and R. R. Salimov: Boundary behavior and the Dirichlet problem for the Beltrami equations. - Algebra i Analiz 25:4, 2013, 101-124.

[9] Kovtonyuk, D. A., V. I. Ryazanov, R. R. Salimov, and E. A. Sevost'yanov: Boundary behavior of Orlicz-Sobolev classes. - Mat. Zametki 95:4, 2014, 564-576.

[10] Kovtonyuk, D. A., V. I. Ryazanov, R. R. Salimov, and E. A. Sevost'yanov: Toward the theory of the Orlicz-Sobolev classes. - Algebra i Analiz 25:6, 2013, 50-102.

[11] Lomako, T., R. Salimov, and E. Sevost'yanov: On equicontinuity of solutions to the Beltrami equations. - Ann. Univ. Bucharest, Math. Ser. 1:2(LIX), 2010, 263-274.

[12] Martio, O., S. Rickman, and J. VäısÄLÄ: Definitions for quasiregular mappings. - Ann. Acad. Sci. Fenn. Ser. A I Math. 448, 1969, 1-40.

[13] Martio, O., V. Ryazanov, U. Srebro, and E. Yakubov: To the theory of $Q$-homeomorphisms. - Dokl. Akad. Nauk 381:1, 2001, 20-22 (in Russian).

[14] Martio, O., V. Ryazanov, U. Srebro, and E. Yakubov: BMO-quasiconformal mappings and $Q$-homeomorphisms in space. - Reports Dept. Math. Univ. Helsinki 288, 2001, 1-24.

[15] Martio, O., V. Ryazanov, U. Srebro, and E. Yakubov: Q-homeomorphisms. - Contemp. Math. 364, 2004, 193-203.

[16] Martio, O., V. Ryazanov, U. Srebro, and E. Yakubov: On $Q$-homeomorphisms. - Ann. Acad. Sci. Fenn. Math. 30, 2005, 49-69.

[17] Martio, O., V. Ryazanov, U. Srebro, and E. Yakubov: Moduli in modern mapping theory. - Springer, New York, 2009.

[18] Rickman, S.: Quasiregular mappings. - Springer-Verlag, Berlin, 1993.

[19] SAKs, S.: Theory of the integral. - Dover, New York, 1964.

[20] Salimov, R.: ACL and differentiability of $Q$-homeomorphisms. - Ann. Acad. Sci. Fenn. Math. 33, 2008, 295-301.

[21] Salimov, R. R., and E. A. Sevost'yanov: ACL and differentiability of open discrete ring ( $p, Q)$-mappings. - Mat. Stud. 35:1, 2011, 28-36.

[22] Salimov, R. R., and E. A. Sevost'Yanov: The theory of shell-based $Q$-mappings in geometric function theory. - Mat. Sb. 201:6, 2010, 131-158.

[23] VÄISÄLÄ, J.: Lectures on $n$-dimensional quasiconformal mappings. - Lecture Notes in Math. 229, Springer-Verlag, Berlin, 1971.

[24] VäıSÄLÄ, J.: Two new characterizations for quasiconformality. - Ann. Acad. Sci. Fenn. Ser. A I Math. 362, 1965, 1-12.

Received 21 March 2014 • Accepted 15 August 2014 